\title{
Development of the ATLAS Liquid Argon Calorimeter Readout Electronics for the HL-LHC
}

Hao Xu, on behalf of the ATLAS Liquid Argon Calorimeter Group

\section{The Liquid Argon Calorimeter}

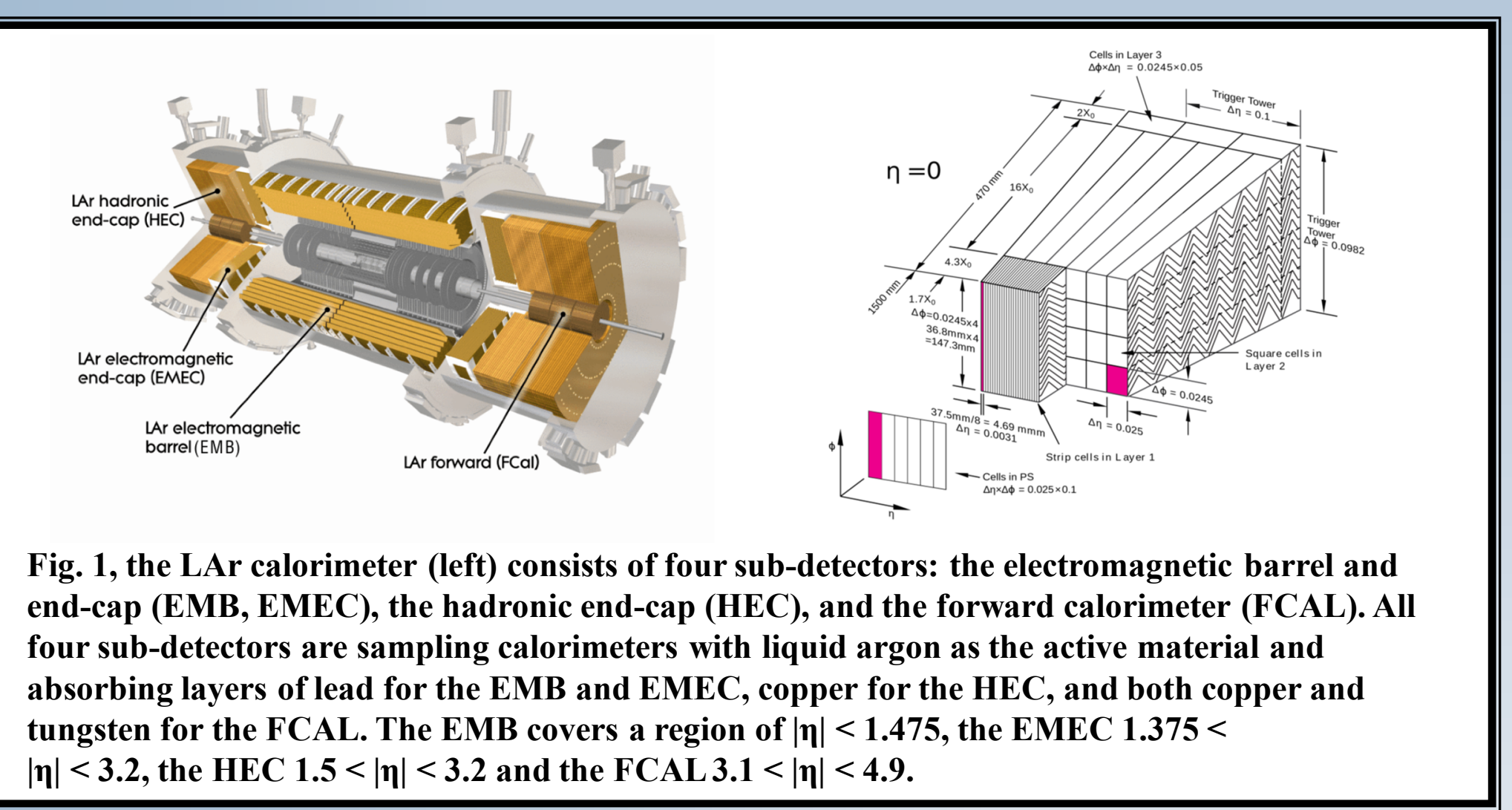

The LHC is scheduled to undergo a major upgrade to reach the ambitious goal of collecting data equivalent to 4 $\mathrm{ab}^{-1}$ of integrated luminosity until 2038. The upgrade will be taken during the long shutdown phase in 2024-2026, then the High-Luminosity LHC (HL-LHC) phase will start. After this point, the peak luminosity is expected to reach a maximum of $75 \mathrm{nb}^{-1} \mathrm{~s}^{-1}$, a value about 4 times higher than today. At the same time, the number of protonproton interactions per bunch crossing is expected to increase by a factor of 4 to 5 , up to a value of 200 . In order to keep up with the increased luminosity, the ATLAS detector will undergo the HL-LHC upgrade, and the Liquid Argon Calorimeter electronics will be replaced to readout the 182,468 calorimeter cells at $40 \mathrm{MHz}$ with 16 bit dynamic range in the high pileup conditions of the high-luminosity LHC. Low-noise, low-power, radiationtolerant and high-bandwidth electronics components are being developed in 65 and $130 \mathrm{~nm}$ CMOS technologies. The off-detector electronics will make use of FPGAs connected through high-speed links to perform energy reconstruction, data reduction and buffering. Results of tests of the first prototypes of front-end components are presented, along with design studies on the performance of the off-detector readout system.

\section{Readout Electronics for HL-LHC}

\section{Electronics requirements:}

- Cover full energy range from electronics noise level to highest possible energy deposited in a single cell: $50 \mathrm{MeV}$ to $\sim 3 \mathrm{TeV}$.

- Linearity of $0.1 \%$ up to $\sim 10 \%$ of the dynamic range (covering electroweak-scale physics).

- Low electronics noise, below intrinsic calorimeter resolution:

- Effective $\sim 11$-bit precision at high energy

- Equivalent precision in analog signal shaping

- All data sent off-detector:

- $1.3 \mathrm{Gbps}$ per channel; $\sim 180 \mathrm{Gbps}$ per frontend board; $\sim 275$ Tbps for the full detector

The architecture of the Phase-II readout is shown in Figure 2. The readout electronics include the on-detector Front-End Boards (FEB2), the off-detector LAr Signal Processors (LASP).

\section{Front-end Electronics}
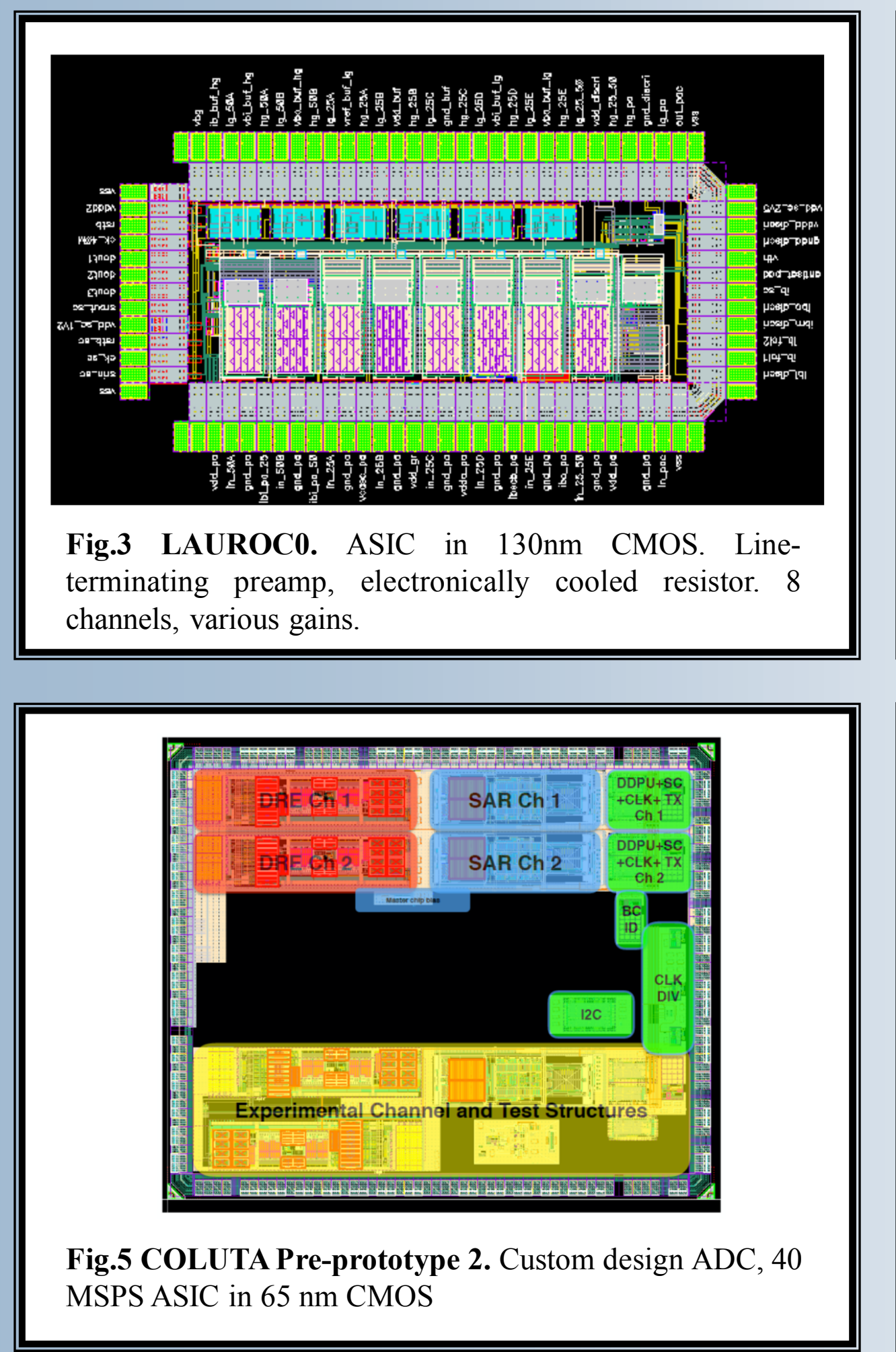

\section{Off-detector Electronics}

\section{The LASP processes digitized waveforms, selects a} gain, implements digital filtering algorithm, interfaces with the trigger and data acquisition system (DAQ), and buffers the data while awaiting a trigger decision. The LASP board design is based on the ACTA standard. Each LASP FPGA takes inputs

Digital filtering algorithms, implemented via FPGAs, process the sampled waveforms to extract energy and timing information. The Optimal Filter (OF) algorithm is currently used by the LAr calorimeter, but to deal
with the increased pileup conditions more sophisticated algorithms capable of out-of-time pileup correction are also under study.

smart Rear Transition Module (RTM) is being developed erial optical links to the trigger $F$

serial optical links to the trigger Feature Extractors (FEX)
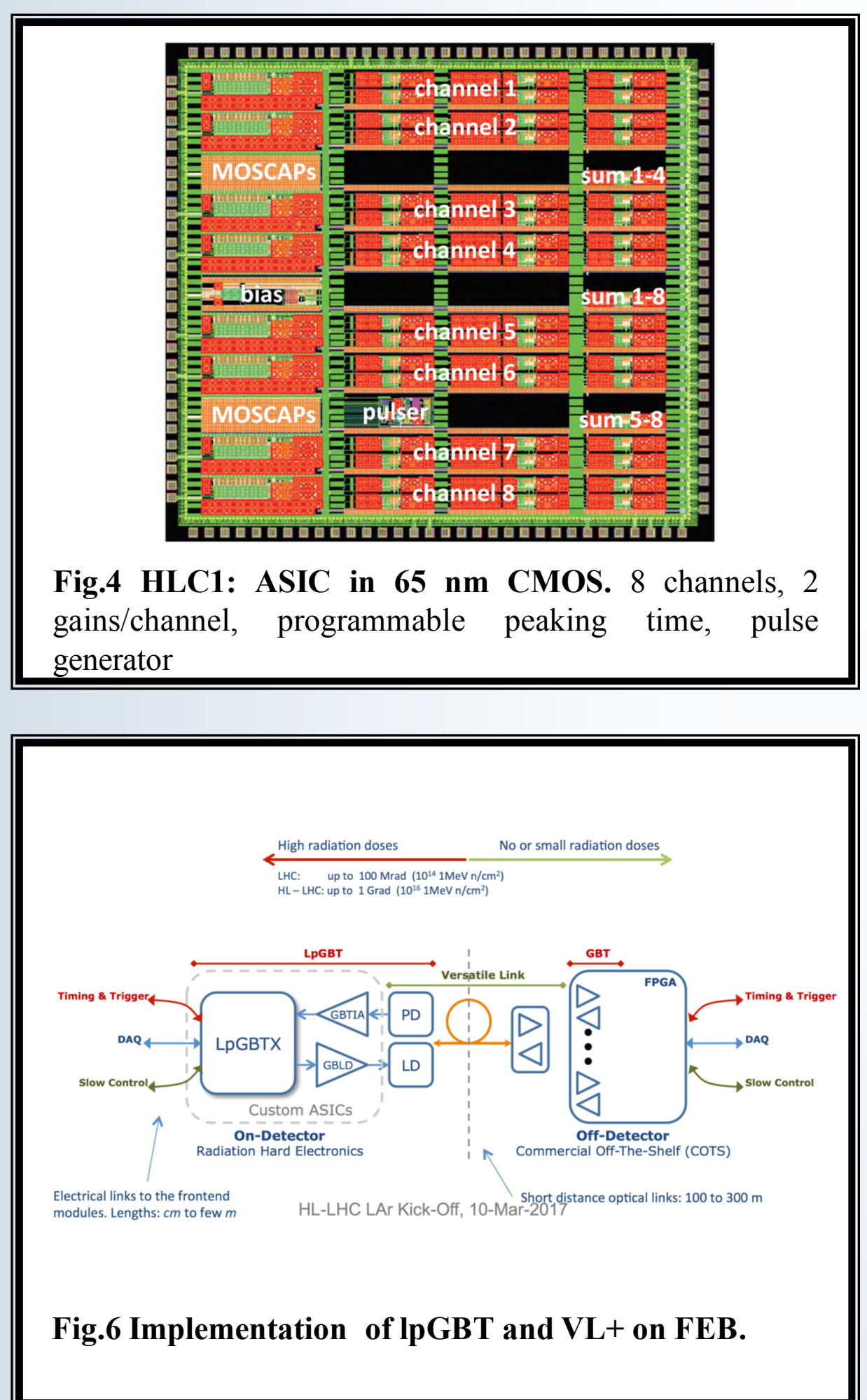

and the DAQ system at transmission speeds between $10 \mathrm{Gbps}$ and $25 \mathrm{Gbps}$. It also allows an implementation of data concentration as well as monitoring and control tasks, thereby enhancing the monitoring and control tasks, the
flexibility of the LASP system.

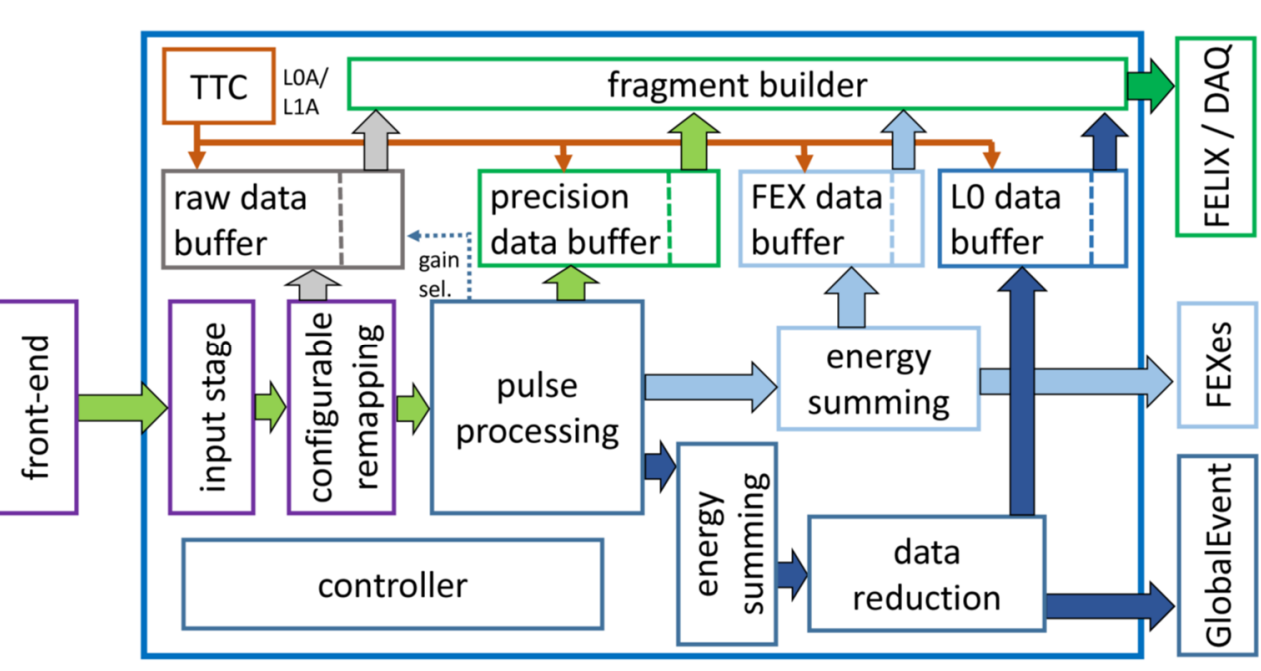

Fig.7 Block diagram of the LASP. Each LASP FPGA receives data from 4 FEB2 boards on 88 links at $\sim 10$ Gbps each and needs

The front-end board (FEB2) is responsible for signal amplification, pulse shaping, digitization, and transmission to the off-detector electronics. Each FEB2 handles 128 channels with three custom ASICs - the Preamplifier-shaper, the Analog to Digital Converter (ADC) and lpGBT

Preamplification and shaping are implemented on a single ASIC. Two prototypes have been constructed and tested. The first design LAUROC0 uses $130 \mathrm{~nm}$ CMOS with a low noise, line erminating preamplifier. The second design HLC1 uses $65 \mathrm{~nm}$ CMOS with a fully differentia mA. For the Phase-II upgrade, the two designs will be merged into a $130 \mathrm{~nm}$ chip.

The cell signal will be digitized at the bunch crossing rate by $40 \mathrm{MHz} 14$ bit and radiation hard COLUTA ADCs. The COLUTA ADC is custom designed in $65 \mathrm{~nm}$ CMOS. Each ADC ASIC consists of a 12 bit SAR, and an additional 2 bit dynamic range enhancer block. The first version of COLUTA has been tested and version 2 has been fabricated and being evaluated in the lab. The readout must cover a wide range of cell energies from the energy of a minimum ionizing particle (MIP) to the maximum cell energies expected for hypothetical $\mathrm{TeV}$ scale particles. In addition, the least significant bit (LSB) of the ADC must be smaller than the electronic noise. As a result, covering the full range of energies requires a dynamic range of 16 bits, which can be achieved using a two gain system.

Data from each FEB2 is transmitted using 22 radiation tolerant optical links $\sim 10 \mathrm{Gbps}$. The lpGBT and $\mathrm{VL}+$ that are developed in CERN-based projects are planned to be used on FEB2.

The power distribution will be upgraded as well to allow possibility of more accessible locations. he LVPS will be replaced with $280 \mathrm{~V}$ DC to $1-4 \mathrm{~V}$ device with new, intermediate $\sim 48 \mathrm{~V}$ step. Radiation-hard, qualified converters are under study.

Performance Study

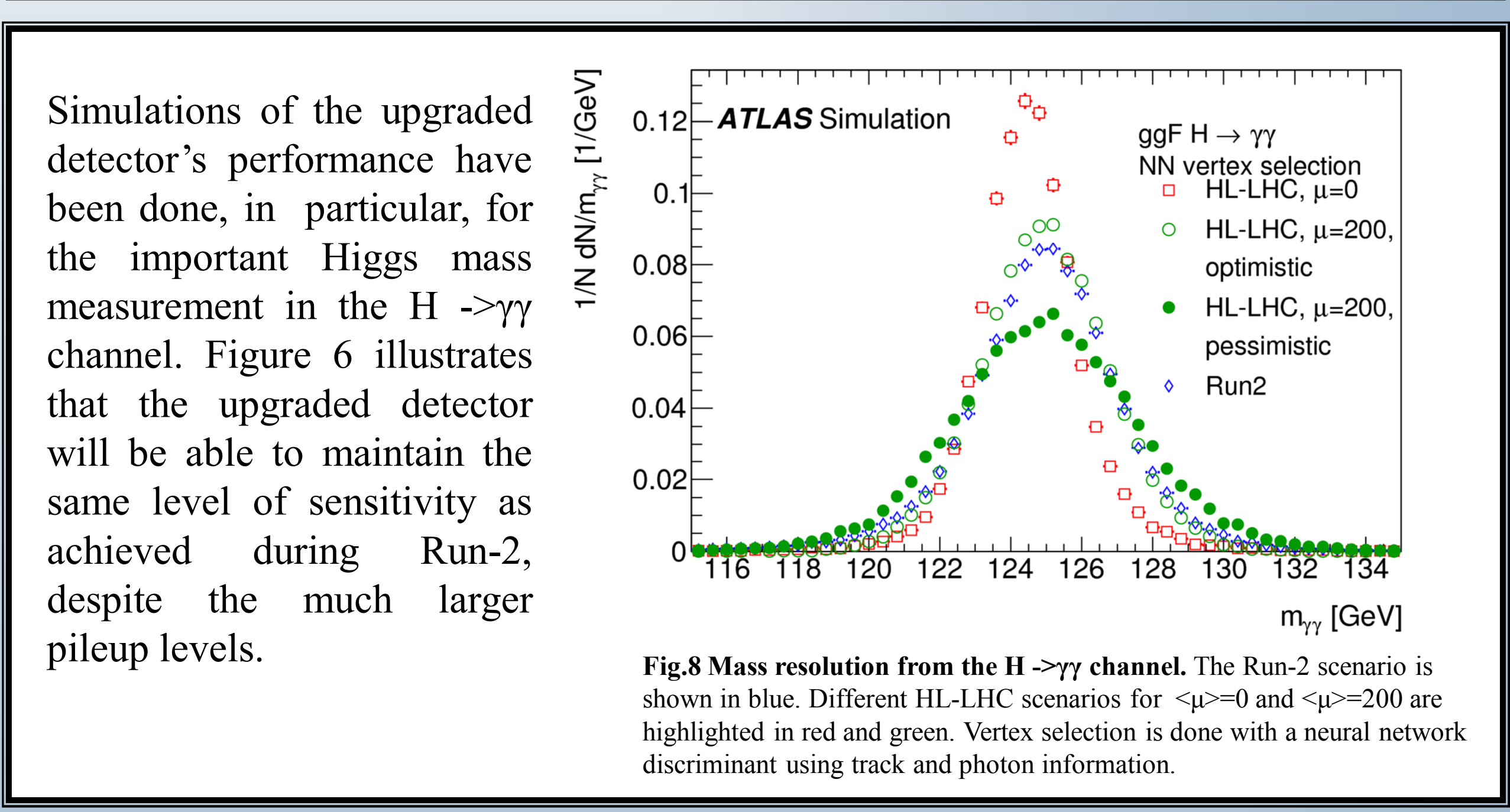

Summary

The LAr Calorimeter will provide data for all of ATLAS physics throughout the HL-LHC. For the Phase-II upgrades, R\&D for a full replacement of the LAr readout electronics is in progress. These upgrades are necessary due to the increased radiation damage and for compatibility with the new trigger system. 